\title{
Role of Magnetic Resonance Imaging in Detection and Follow up of Typical/atypical Posterior Reversible Encephalopathy Syndrome "PRES" in Pediatric Cancer Patients
}

\author{
Medhat M. Refaat ${ }^{\text {a }}$, Iman M. Zaky ${ }^{\text {b }}$, Islam M. El Shazly ${ }^{\text {a }}$, Mostafa M. Abd Al Gawad ${ }^{\text {a }}$
}

\begin{abstract}
a Department of radiology, Benha faculty of medicine, Benha University, Egypt. b Department of radio-diagnosis national cancer institution, Cairo university, Egypt.
\end{abstract}

Correspondence to: Mostafa M. Abd Al Gawad, Department of radiology, Benha faculty of medicine, Benha University, Egypt

Email:

mostafaabdelgawad85@gmail.com

Received: 28 March 2020

Accepted: 18 April 2020

\begin{abstract}
:
Purpose: to evaluate current role of magnetic resonance imaging in diagnosis, characterization and follow up of pres in pediatric cancer patients. Methods: we identified 50 cases of children diagnosed with extra cranial cancer with cns manifestations suspecting pres. patients presented to radiology department in (57357-cche) 2013-2015. Results: there was slightly male predominance $(56 \%) .62 \%$ of patients were less than 10 years of age with median age 8.5 years. pres developed in $74 \%$ of patients within less than 6 months from the start of treatment while $26 \%$ developed it more than 6 months. primary diagnoses were leukemia $(n=36)$, lymphoma $(n=9)$, neuroblastoma $(n=3)$ and post bmt $(n=2)$. convulsions were the most frequent presenting symptom (78\%) of the patients, ams (34\%), headache (26\%) and visual impairment occurred in $6 \%$ of studied patients. mri study revealed that atypical pres $(62 \%)$ was more common than typical type with posteriorly dominant disease noted in 45 cases, 7 cases showed restricted diffusion and 4 cases showed post contrast enhancement. 41 patients underwent follow up mri
\end{abstract} study, where, 23 of them showed complete radiological resolution, 10 patients showed regressive course while 7 cases showed progressive course. Conclusions: pres is more common in children with hematological malignancies with reversible radiologic outcomes in most of the cases yet small number of patients will develop persistent brain damage. mri is the gold standard for diagnosing pres. atypical pres, areas of true restricted diffusion or post contrast enhancement showed higher incidence of poor prognosis.

Keywords: Children cancer patients; posterior reversible encephalopathy syndrome (PRES); magnetic resonance imaging (MRI) 


\section{Introduction}

Childhood cancers are rare, representing less than $1 \%$ of new cancer diagnoses; however, they are still the leading cause of disease-related death in children (1).

In the past, central nervous system complication of cancer therapy was rare because diseases were almost rabidly fatal. More recently advances in imaging technique and treatment methods have improved the prognosis and prolonged the survival rate; however, the frequency of complication central nervous system has increased (2).

Central neurotoxicity constitutes a major clinical concern in cancer pediatric patients. Acute neurotoxicity is associated with the intensification of chemotherapy and has been reported in approximately $10-20 \%$ of leukemia patients; however, some studies have reported treatment-related abnormalities detectable by MRI in up to $20 \%$ of patients, which indicates that neurotoxicity events may be underreported (3).

Posterior reversible encephalopathy syndrome "PRES" is one of the side effects of chemotherapy and bone marrow transplantation which are frequently used in treatment of most common pediatric cancers leukemias and neuroblastoma (4).
Posterior reversible encephalopathy syndrome is a clinic-neuroradiological disease entity represented by characteristic MR imaging, most often in the parietooccipital lobes accompanied by clinical neurological alteration ranging from headache, altered mental status, vision loss and convulsion to loss of consciousness (5). It was described for the first time by Hinchey et al in 1996 (6).

Although the pathophysiology of PRES remains unknown, the currently preferred explanation is related to hypertension, impaired auto regulation and hyper perfusion (7).

\section{Aim of the work}

To evaluate current role of magnetic resonance imaging in diagnosis, characterization and follow up of posterior reversible encephalopathy syndrome in pediatric cancer patients.

\section{Patients and Methods}

Type of the study: original article

Subjects:

This study included 50 children diagnosed with extra cranial cancer on or after chemotherapy and/or bone marrow transplantation with CNS manifestations. 
All the patients presented to radiology department in Children's Cancer Hospital Egypt (57357-CCHE) in the time between November 2013 to December 2015. The study was approved by the institutional ethics committee.

\section{* Inclusion criteria:}

Children diagnosed with extra cranial cancer on or after chemotherapy and/or bone marrow transplantation represented with acute onset of neurological manifestation including headache, visual disturbance, disturbed consciousness or seizure with or without increased blood pressure.

\section{* Exclusion criteria:}

1. Patients older than 18 years old.

2. Patients with contraindication to MRI: e.g.: Pacemaker \& metallic implant

3. Patients with contraindication to contrast: Patients with disturbed renal function test (if creatinine>2), patients with glomerular filtration rate $<30 \mathrm{ml}$ per min. per $1.73 \mathrm{~m} 2$ or any acute renal insufficiency related to the hepato-renal syndrome or perioperative liver transplantation.

4. Patients with primary CNS tumor.

5. Patients with metastatic brain tumor.

6. Patient with history of cranial radiation
- Patient preparation:

Detachable metallic implants like teeth prosthesis are considered as proportional contraindication and should be removed prior to entrance to magnetic area.

As patients will need anaesthesia, fasting 46 hours before the scan is required.

\section{MRI Technique: -}

\section{Conventional MRI (MRI):}

All patients were evaluated by limited MRI technique using 1.5 Tesla unit (Siemens Espree) and 3 Tesla (Philips, Ingenia) MRI scanners medical Systems.

All the cases were examined in supine position with standard circularly polarized head coil.

\section{The used protocol basically consists of} axial T1WI, T2WI \& FALIR images with sagittal T1WI image .DWI and corresponding ADC maps done for all cases .Selected cases received intravenous administration of Gadolinium- DTPA (o. 2 $\mathrm{mmol} / \mathrm{kg}$ ), contrast enhanced T1WI in axial, sagittal and coronal planes are obtained as well as MR Venography is added according to the clinical suspicion.

\section{Results}

According to demographic data, this study includes 50 cancer patients; they developed acute neurological manifestation suggestive 
of PRES (28 male and 22 female) with male: female ratio $1: 1.27$.

\section{Patient and causes -related Characteristics:}

Among the 50 patients with PRES, 31 were $<10$ years of age $(62 \%)$, while 19 patients were $\geq 10$ years of age (38\%) with mean age 8.5 years and range from 11 months to 17 years old. PRES was the most common in leukemia and lymphoma in 45 patients $(90 \%)$ followed by $\mathrm{NB}$ in 3 patients $(6 \%)$ and post bone marrow transplantation in 2 patients $(4 \%)$. The time for PRES development from starts of treatment ranging from 0.03 to 18 months, with average 4 months, 37 patients (74\%) developed it in less than 6 months while 13 patients $(26 \%)$ developed it more than 6 months from the start of the treatment. Forty patients (80\%) received chemotherapy or immunosuppression for 14 days with mean of 14.4 days with standard deviation 30.09. While 10 patients (20\%) developed the clinical findings after 2 weeks from the last chemotherapy and had other co morbidities and risk factors ( 7 patients were hypertensive, one patient had typhlitis with sepsis and multi organ system failure, one patient had sepsis and septic shock while the remaining patient hasn't any identified risk factor or comorbidity).

\section{Clinical Presentation}

In our study, the most common presenting symptom was seizures that was present in 39 out of 50 (78\%), followed by severe altered mental status that presented in 17 out of $50(34 \%)$ and persistent headache, that was present in 13 out of 50 (26\%) patients. The other associated symptoms were visual deficit ( 3 out of $50,6 \%$ ) \& facial palsy (just one case each) (2\% each).

\section{Radiological finding:}

\section{Location of PRES:}

In our study, the most commonly involved locations were the parieto-occipital lobes (45 out of 50) (90\%). These locations are considered the typical distribution of PRES.

The other affected regions were frontal lobe $(21 / 50) 42 \%$, the posterior fossa $(5 / 50)$ $10 \%$, temporal lobe $(11 / 50) 22 \%$, basal ganglia (2/50) 4\%, thalamus (3/50) $6 \%$, and white matter $(2 / 50) 4 \%$. These regions are considered the atypical distribution of PRES.

Forty- eight patients showed bilateral distribution of the lesion classified as $60.4 \%$ (29) were asymmetrical \& 39.6\% (19) were symmetrical while only two cases were unilateral. 
$>$ Typical versus atypical MRI findings:

We consider the PRES to be typical if the MRI shows symmetrical distribution of vasogenic edema, predominantly within the parieto-occipital regions, with no evidence of diffusion restriction, hemorrhage or enhancement. It represents $38 \%$ (19 out of 50) in our study.

The atypical PRES represents $62 \%$ (31 out of 50) in our study, being classified as atypical location \& atypical presentation.

The atypical location is considered if there is involvement of any area of the brain unless the parieto- occipital regions. It represents $87.1 \%$ (27/31) in our study.

The atypical presentation is considered if there is asymmetrical distribution of the lesion, contrast enhancement, restricted diffusion or hemorrhage.

The most common atypical criteria of PRES presented in our study is asymmetrical distribution of the lesion which is found in $93.54 \% \quad$ (29 out of 31), followed by diffusion restriction which is found in $22.58 \%$ (7 out of 31) cases, then contrast enhancement which is depicted in $12.9 \%$ (4 out of 31 ) cases with no hemorrhage seen in our scanned cases.

\section{> Grading of PRES:}

Grading of PRES depends on the extension of brain edema within the brain parenchyma especially on T2 /FLAIR images. Patients are divided into 4 grades where $6 \%, 32 \%, 40 \%$ and $22 \%$ were with grade I, II, III and IV respectively. (table $1)$.

Table No.1: Grading severity of PRES

\begin{tabular}{|c|c|c|c|}
\hline $\begin{array}{c}\text { Minimal } \\
\text { (Grade 1) }\end{array}$ & $\begin{array}{c}\text { Mild } \\
\text { (Grade 2) }\end{array}$ & $\begin{array}{l}\text { Moderate } \\
\text { (Grade 3) }\end{array}$ & $\begin{array}{c}\text { Severe } \\
\text { (Grade 4) }\end{array}$ \\
\hline $\begin{array}{c}\text { Involveme } \\
\text { nt of one } \\
\text { lobe }\end{array}$ & $\begin{array}{c}\text { Involvement } \\
\text { of } 2 \text { lobes }\end{array}$ & $\begin{array}{l}\text { Involvemen } \\
t \text { of } 3 \text { lobes }\end{array}$ & $\begin{array}{l}\text { Involvement of } \\
\text { all lobes from } \\
\text { the ventricular } \\
\text { margin to the } \\
\text { subcortical } \\
\text { white matter, or } \\
\text { involvement of } \\
\text { corpus callosum, } \\
\text { basal ganglia, } \\
\text { thalami, or } \\
\text { internal } \\
\text { capsules. }\end{array}$ \\
\hline
\end{tabular}

\section{Follow up MRI:}

Eighty two percent (41 out of 50) of the patients were followed up by MRI and $18 \%$ (9 out of 50) didn't.

Out of the 41 patients who underwent MRI follow up, $56.1 \%$ (23) showed complete resolution and $24.39 \%$ (10) showed regressive course of the disease, 7 cases (17.07\%) showed progressive course and just one case $(2.43 \%)$ showed mixed course.

\section{$>$ Relation between Grading and Sequelae of PRES:}

Patients with PRES were divided according to their sequelae into 2 groups: Group A with complete resolution or significant 
regressive course of the radiological manifestations of PRES and group $B$ with stationary or progressive course of the manifestations.

Thirty three out of forty one patients $(80.48 \%)$ that underwent follow up by MRI showed complete resolution or significant regressive course and were classified as group A. 8 out of 41 patients $(19.51 \%)$ showed stationary course \& progressive course and were classified as group B

Eight out of 41 patients that underwent follow up by MRI and were classified as group B had severe, extensive, and confluent involvement of 3 or all lobes, or involvement of the following: $\mathrm{BG}$, thalami or extensive involvement of the posterior fossa (Grade 4); all of them showed progressive disease or have stationary course.

Thirty three out of forty one patients that underwent follow up MRI which are classified as group A showing minimal (Grade 1), mild (Grade 2) and moderate (Grade 3) grade of involvement; $80.84 \%$ shows complete resolution or significant regressive course (Group A).

This study shows that the sequelae of patients with grade 4 had the worst significant prognosis than the sequel of patients with grade 1, 2 and 3, although no significant difference was found between the first three grades.

Relation between diffusion restriction $\&$ enhancement to Sequelae of PRES:

\section{I- DWI restriction:}

In our study (7 out of 50) $14 \%$ of the patients showed restricted diffusion, indicating progression to ischemic infarction. Only 5 patients of them underwent follow up by MRI, two of them showed regressive course of the disease however, the other three cases showed progressive or mixed course of the disease.

\section{II-Contrast enhancement:}

In our study (4 out of 50) $8 \%$ showed variable degrees of contrast enhancement, all cases underwent follow up by MRI; three of them showed progressive course of the disease while only one case showed regressive course.

\section{DISCUSSION}

Posterior reversible encephalopathy syndrome is more common in children with hematological malignancies compared with other tumors and is associated with hypertension, chemotherapy and steroid use. The outcome is favorable in most of the cases and symptoms resolving within few days. However, a small number of cases will develop persistent brain imaging changes and neurological deficit (8). 
Although PRES has gained increasing attention since its description in 1996 and much has been learned in terms of the various clinical and imaging characteristics of the syndrome, data on outcome from larger studies are still limited (9).

Such information might be valuable for physicians who are involved in the care of pediatric cancer patients with PRES to guide prognostication and therapy. For this reason, we thought to determine factors associated with good outcome and morbidity in this category of patients. The clinical and imaging characteristics of our PRES study were compared to previously published studies.

This study addresses the risk factors, clinical, radiological outcome and prognostic factors among 50 pediatric patients with childhood cancer who developed PRES during the period from November 2013 to December 2015 at Children's Cancer Hospital Egypt (57357CCHE).

In accordance with the recent literature, our study shows that PRES is a heterogeneous syndrome with various imaging features and clinical presentation $(10 ; 11)$.

In our study, PRES occurred in a wide range of malignancies and predisposing conditions ranging from exposure to various immunosuppressant and cytostatic drugs, hypertension, bone marrow transplantation or sepsis

Regarding patients' characteristics, we found that there was slightly male predominance (56\% were males). Of the whole patients $62 \%$ were less than 10 years of age at the time of PRES development with median age 8.5 years and ranging from 11 months to 17 years old. Khan et al reported a similar age of 9 years and ranging from 1 to 17.5 years old (8). Also, a large pediatric study done on 112 pediatric cancer patients, Zama et al found the average age at the time of PRES was 9.2 years (range 1-17) (11).

In the current study, the time for PRES development from the start of chemotherapy ranged from 0 to 30 months, with average 4 months, (74\%) developed PRES with a time less than 6 months from the start of treatment while $(26 \%)$ were more than 6 months. While Khan et al study found that the median time for PRES development from the start of chemotherapy is 6.6 months and ranging from 0 to 101.4 months $(\mathbf{8})$.

PRES was most common in hematological malignancies that represents $(72 \%)$ of the patients who developed PRES in this study divided into (60\%) had acute lymphoblastic leukemia and (12\%) had acute myeloid leukemia. PRES was more frequently observed during induction phase of therapy. 
Seconded most common primary disease in our study was lymphoma that represented $(18 \%)$ of our patients. Khan et al study found that $67.5 \%$ of patient developed PRES had hematological malignancies with ALL represents (43\%) (8) and Zama et al found primary hematological malignancy in $72.3 \%$ of patients developed PRES with $(53.5 \%)$ of them were ALL (11).

While investigating the risk factors related to posterior reversible encephalopathy syndrome (PRES) we found that hypertension, chemotherapy and steroid administration within the last 14 days were the most common related factors for the development of PRES in our patients. Also, Khan et al found that $94.5 \%$ had hypertension, a steroid used in $78.3 \%$ of patients and $56 \%$ of the patients had received chemotherapy within last 14 days prior to PRES development (8).

In this study, sepsis was present in 2 patients (4\%), this was in agreement with Hinduja et al, in which sepsis was present in $7 \%$ of the cases (10). Masetti et al. assumed that sepsis and septic shock seems to have a role in the pathogenesis of PRES development by two mechanisms: endothelial damage and microcirculation disturbances (12).

As regards clinical presentation, convulsions were the most frequent presenting symptom, it was found in $78 \%$ of the patients. Similarly, convulsions occurred in $97 \%$ and $86.6 \%$ respectively of studied pediatric patients $(8 ; 11)$. While in contrast with the study of 100 adult patients by Hinduja et al., convulsions presented in $58 \%$ of patients (10). This may be due to that the occurrence of convulsions being more common in pediatrics than adults in PRES (13).

Altered of mental status (AMS) occurred in $34 \%$, compared to $67 \%(8)$ and $59 \%$ in other study (14). While a headache was present in $26 \%$ of studied patients, Schweitzer et al reported a higher frequency of $54 \%$ in adult patients (15). In this study, visual impairment occurred in $6 \%$. A frequency comparable to previous studies(14;10).

Regarding the radiological findings, in the present study, atypical PRES was more common than typical type where it was found in $62 \%$, while typical PRES was found in $38 \%$ of the patients. This was consistent with previous study where the atypical pattern was predominate $(61 \%)$ (14), while another study showed that the typical PRES pattern was found in $80 \%$ (16).

A posteriorly dominant disease was noted in almost all the studied cases (90\%) (fig no. 1), similar results have been reported previously $(17 ; 18)$. Till now, it is not completely understood why does the 
syndrome tends to dominate in the posterior regions, however, it is thought that this dominance is related to the presence of a lesser sympathetic supply over the posterior circulation in comparison with the anterior cerebral circulation, thus the regulatory mechanisms that helps preserving the brain tissue in the presence of hypertensive insults are less effective $(19 ; 20)$.

In our study, frontal lobe involvement is seen in (42\%) of our cases ( fig 2) compared to $(25 \%)$ reported previously by other colleagues (18).

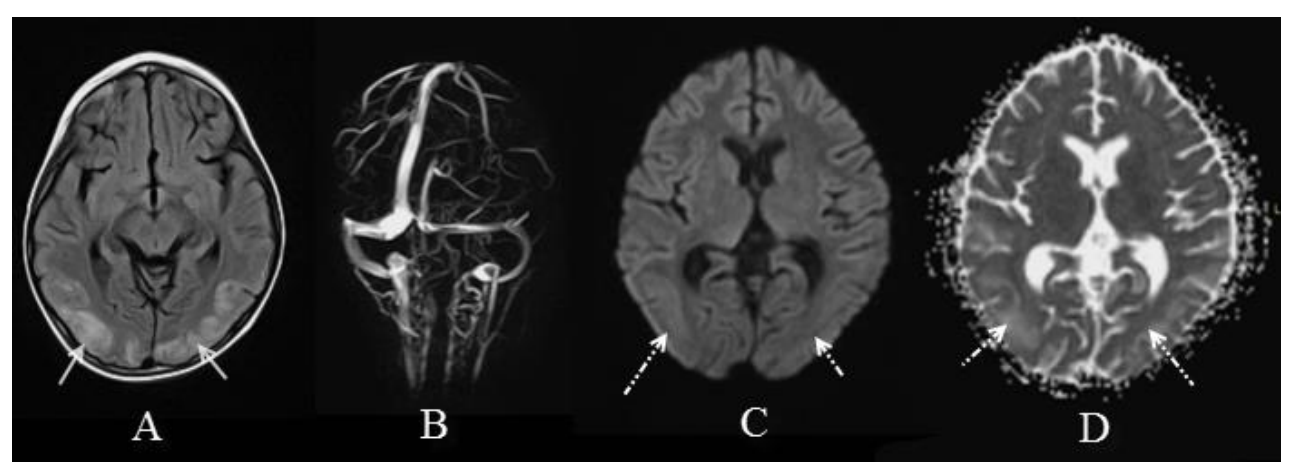

(Fig no. 1): An axial FLAIR image (A) demonstrating a bilateral occipito-parietal involvement (arrows). MRV image (B) showing no gross abnormality. Diffusion image (C) and the corresponding ADC map (D) showing areas of facilitated diffusion at the occipito-parietal region bilaterally (dotted arrows).

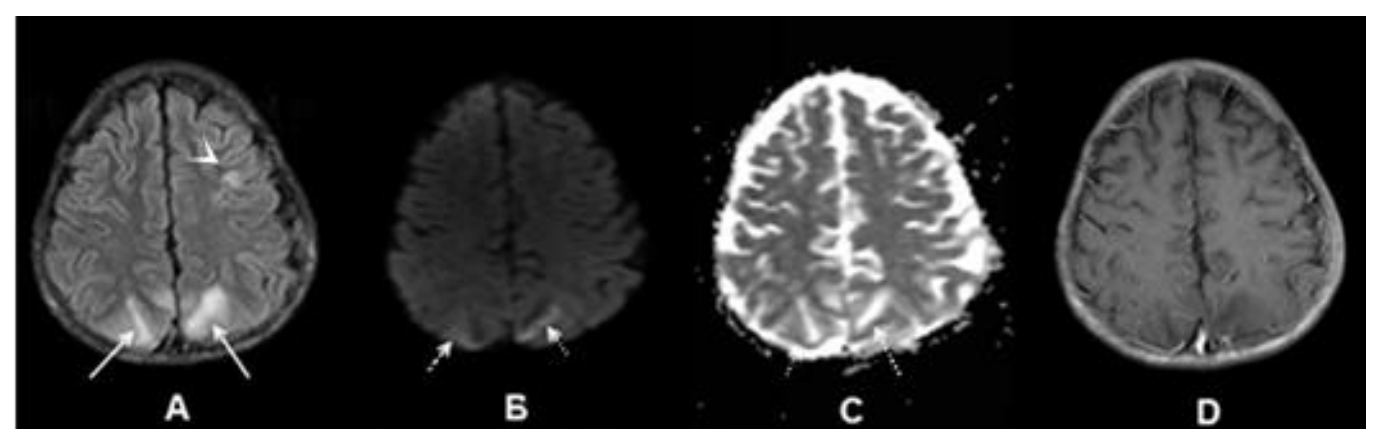

(Fig no.2): axial FLAIR sequence image (A) demonstrating bilateral parieto-occipital (arrows) as well as deep left frontal lobe (arrow head) involvement. axial diffusion images (B) and the corresponding ADC map (C) showing a linear areas of true restriction (dotted arrows) adjacent to area of facilitated diffusion

Percentages of temporal lobe involvement

(fig 3) in other studies are much diverse, they have ranged from $33 \%$ (21) to total involvement in all the patients (17), we have reported $22 \%$ temporal affection in our study. Less common areas of involvement included the posterior fossa
(10\%) (fig 4), thalamus (6\%) (fig 5) and basal ganglia (lentiform or caudate) (4\%) were detected in the current study along with previous studies $(\mathbf{8 ; 1 8 )}$. No cases involved only the orbitofrontal region.

The patterns of PRES identified in our study were in form of "Holo-hemispheric 
watershed pattern", it's noticed in $28 \%$ of cases (fig no.6) compared to $23 \%$ reported by Bartynski. $42 \%$ of the cases were expressing the "Dominant parieto-occipital pattern" (fig no.2) (compared to 22\%), whereas $16 \%$ were expressing the "Superior frontal sulcus pattern" (fig no.7) and 12\% expressing "Partial or asymmetric expression" (fig no.8) compared to $28 \%$ noted by Bartynski (22) yet the least expression was the central PRES-variant of the basal ganglia representing $4 \%$.

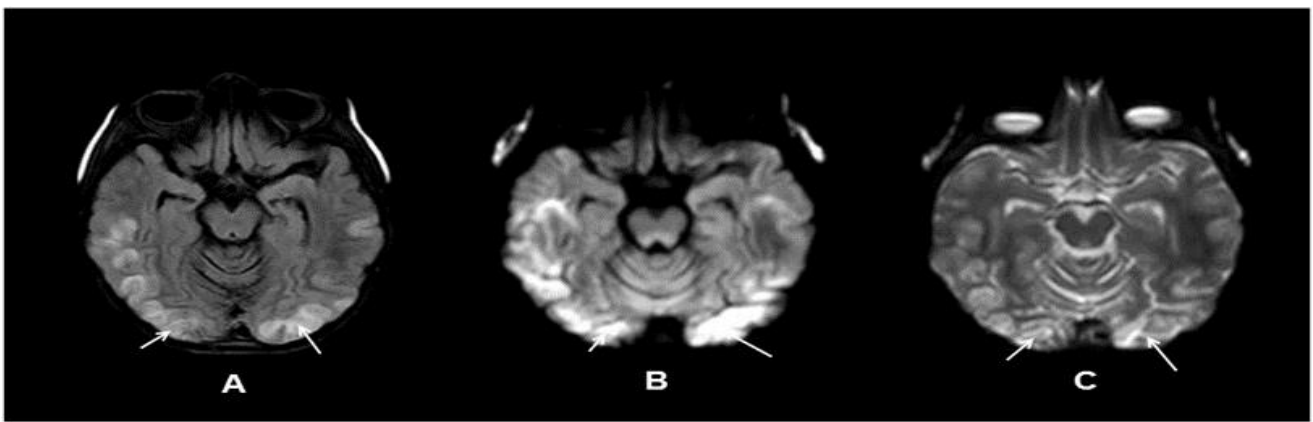

(Fig no.3): Axial FLAIR sequence images (A) demonstrating bilateral temporal high signal (arrows). Axial diffusion images (B) and the corresponding ADC maps (C) at same level showing no gross areas of DWIrestriction.

$\underline{\text { (fig }}$

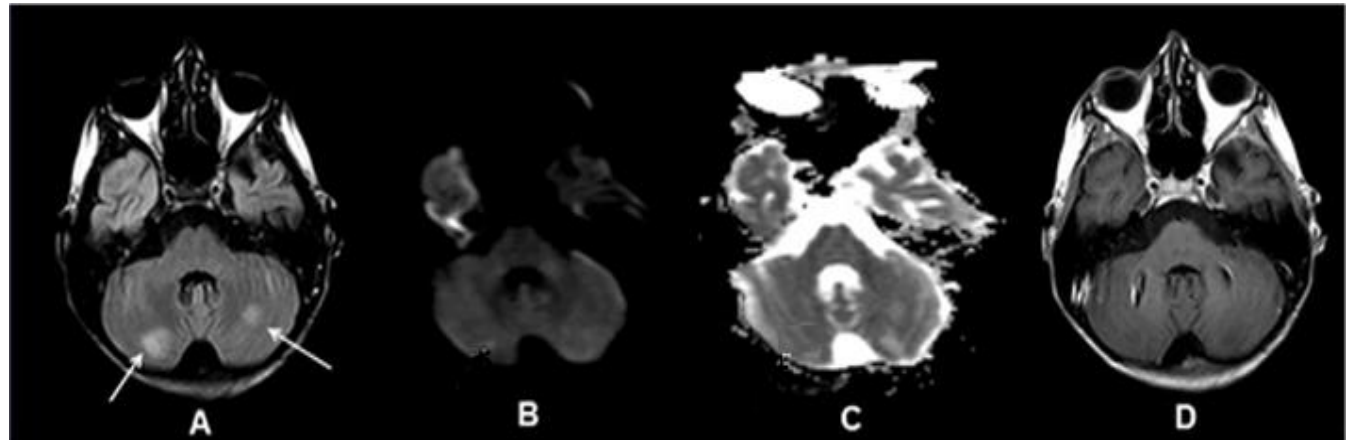

no.4): axial FLAIR sequence image (A) demonstrating bilateral cerebellar (arrows) involvement. Axial Diffusion image (B) and its corresponding ADC map (C) as well as post-contrast T1-weighted image (D) showed no significant diffusion restriction or post contrast uptake respectively

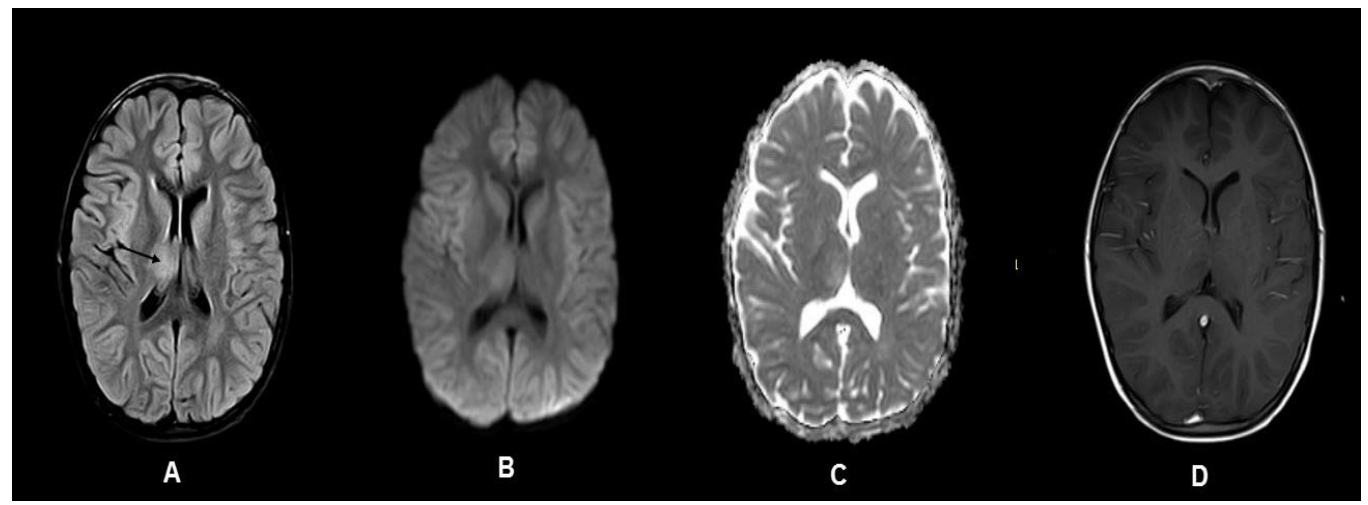

(fig no.5): An axial FLAIR sequence image (A) demonstrating the right thalamic involvement with area of high signal \& subtle mass effect (black arrow). Diffusion image (B) and its corresponding ADC map (C) as well as post-contrast T1-weighted image (D) showed no significant diffusion restriction or post contrast uptake respectively. 


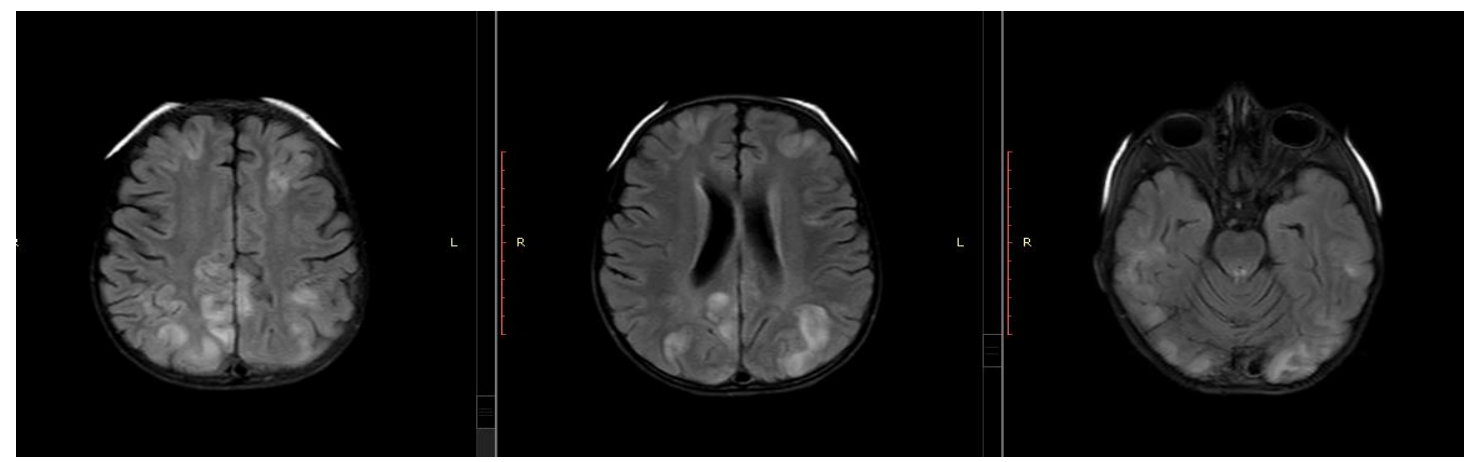

(fig no.6): axial FLAIR image at different levels demonstrating confluent vasogenic edema extending through frontal, parietal occipital as well as temporal lobes involving the watershed zones.

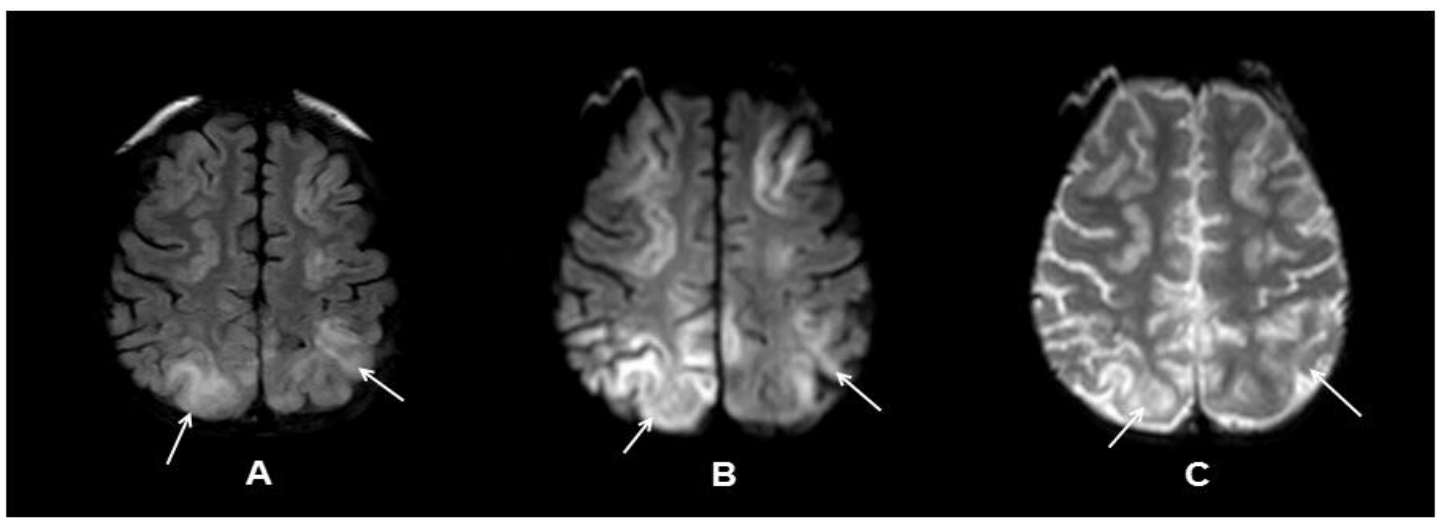

(Fig no.7): Axial FLAIR sequence images (A) demonstrating bilateral superior frontal sulcus and parietal lobes vasogenic edema high signal (arrows). Axial diffusion images (B) and the corresponding ADC maps (C) showing no gross areas of DWI-restriction.

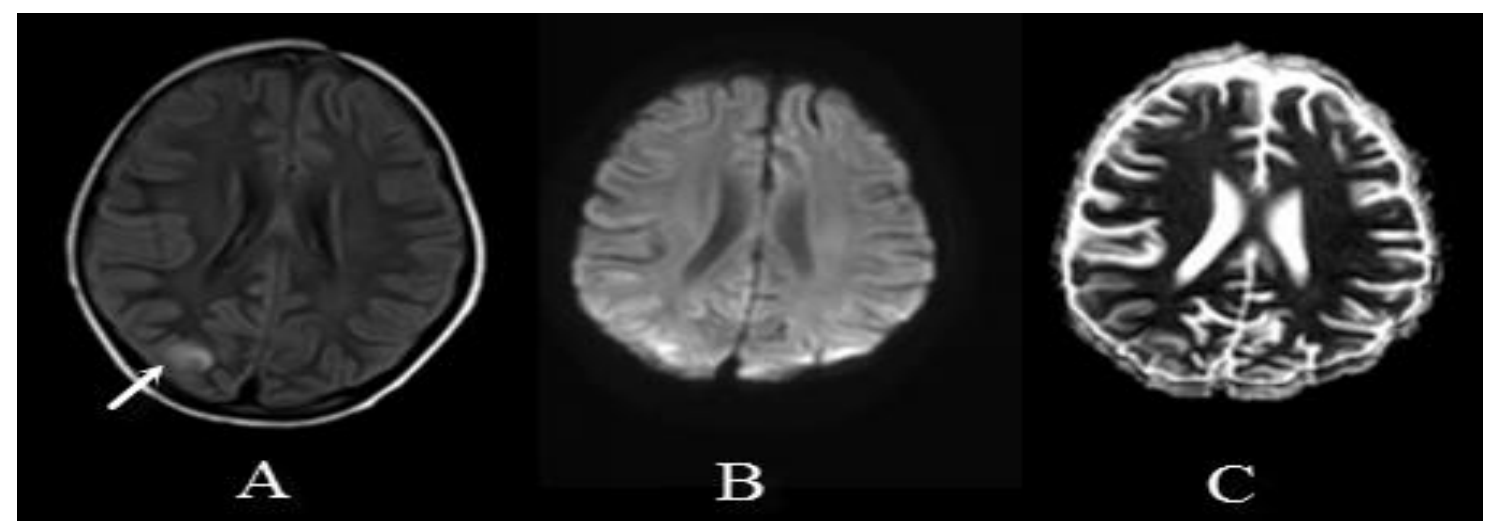

(Fig no.8): An axial FLAIR sequence image (A), demonstrating right occipital sub-cortical focal involvement (arrow). Diffusion image (B) and the corresponding ADC map (C) showing no corresponding area of diffusion restriction. (Asymmetrical expression pattern of PRES)

It is not well understood why does PRES presents in these different patterns. Probably, being a multi-factorial process is reasonable. Since hypertension is one of the major PRES associations, the presence of an already diseased arteries should be kept in mind in addition to the normal arterial brain anatomic variants, though these factors haven't yet studied (23). 
After the introduction and the increased utility of FLAIR which demonstrated a higher cortical involvement percentage among PRES patients (17). In our study, $98 \%$ of the patients had subcortical lesions, with only one case showing central PRESvariant of the basal ganglia. However, cortical involvement was recognized in $92 \%$ of the studied cases. Though it is easy to explain such a high percentage of cortical involvement theoretically, the finding in fact is much higher than the figures reported in the literature, with no reasonable clinical or radiological explanation (24).

Basal ganglia involvement was seen in 2 patients (4\%) compared to $19 \%$ which was noted by Casey and his colleagues, (17). The described pattern above shouldn't be confused with the central PRES-variant of the basal ganglia, as the later devoid of any cortical or sub-cortical affection (19), which there is only one case in our study.

Whether the PRES lesions are symmetrically or asymmetrically distributed over both hemispheres; lesions should present on both sides in order to fulfill the diagnostic criteria for diagnosing PRES (25). However, atypical PRES cases with a unilateral distribution were also reported (22). In our study, unilateral PRES was seen in two cases in which there is no contralateral affection, the rest of the cases (96\%) showed typical bilateral distribution feature which describe the syndrome. From those bilateral cases, $60.4 \%$ were asymmetrically distributed, compared to $16.7 \%$ which is the range present in the literature (22).

In our study supra-tentorial involvement was recognized in all of the PRES patients included, in other words, no isolated brain stem, pons, spinal cord or cerebellar involvement do present. Nonetheless, associated infra-tentorial involvement was noted in this study, most of which are of cerebellar one $(10 \%)$ comparable to $(33 \%)$ documented in the literature of $(21 ; 22)$.

The severity of PRES was evaluated regard to the extent of edema found. The assessment criteria were first used by Mckinney and his colleagues, who have adopted the criteria with respect to the prognostic reviews available.

In our study advanced PRES was present in $22 \%$ while, Schweitzer found it in $49.5 \%$ of the cases (15) this may be due to that PRES edema is being more severe in adults than pediatrics (13).

As regards the patients' outcome (23 out of 41) $(56.1 \%)$ patients who underwent follow up study showed complete remission ,24.39\% developed regressive course while $19.44 \%$ showed progressive course or persistent brain damage which is in diverse with poor outcomes reported in 
previous studies $(\mathbf{1 4 ; 8 ; 1 0 ; 1 1 ) . ~ T h e y ~}$ performed their study on adult cancer patient as Kamiya-Matsuoka et al reported only a complete recovery rate of $51 \%$ within 8 weeks after the onset of PRES as well as death of (15 out of 69) $21.73 \%$ of patients (14). This was consistent with Siebert et, al which assumed that outcome in pediatrics is much better than adults (13).

Diffusion imaging and ADC maps have demonstrate true restriction within $14 \%$ of the cases (fig $\mathbf{9}-\mathbf{b} \boldsymbol{\&} \mathbf{c}$ ), Hinduja et al showed a similar percentage $18 \%$ (10). This was related to the severity of PRES at presentation; with true restriction was noted to occur only in cases under the "moderate" and "severe" syndrome categories.

Focal and linear contrast enhancement was found in $8 \%$ of the patients (fig no.9-d) which is similar to that documented in literature (10).

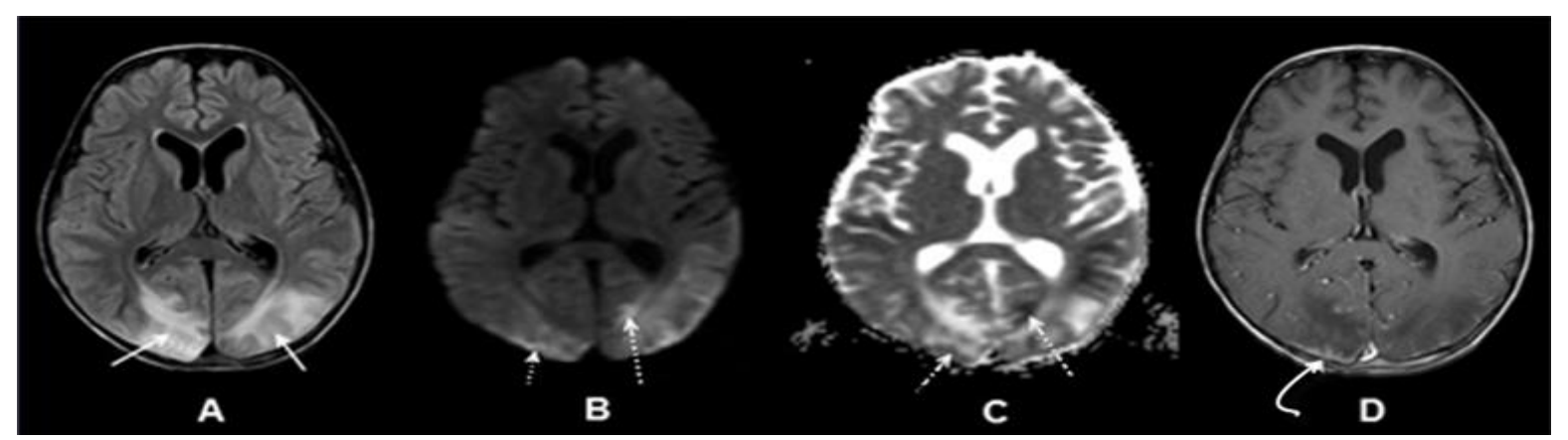

(figno.9): axial FLAIR sequence image (A) demonstrating bilateral parieto-occipital (arrows) cortical/subcortical and deep white matter vasogenic edema. axial diffusion images (B) and the corresponding ADC map (C) showing multiple areas of true diffusion restriction (dotted arrows) adjacent to area of facilitated diffusion. axial T1-post contrast image (D) showing a linear area of cortical enhancement at right occipital lobe (curved arrow).

The term of "Posterior Reversible Encephalopathy Syndrome" may not be accurate since the outcome is not always reversible, the lesions are not always posterior. So, the most recent abbreviation of PRES (Potential Reversible Encephalopathy Syndrome) may be more reliable than the old name.

One of the most important issues related to the outcome of PRES is how to predict the patients who are going to respond well, and not many studies addressed this issue. Only a few studies to our knowledge addressed this $(\mathbf{1 0 ; 1 5 )}$. For this reason, we wanted to study the relationship between the warning signs particularly in radiological finding and outcome of the PRES.

In our study, we found many radiological findings which were associated with poor prognosis. Extensive vasogenic edema, 
frontal lobes, deep whit matter or corpus callosum affection, the presence of large area of restricted diffuse or post contrast enhancement all are considered as warning signs for poor prognosis of PRES. These findings were also depicted by other literature $(10 ; 15)$.

Also, the time from the start of chemotherapy till the development of PRES $\geq 6$ months, patients who had other comorbidities at the time of development of PRES, those who presented with sever generalized convulsions, motor power affection or who had status epilepticus were associated with poor prognosis.

In spite of PRES is a rare syndrome occurred in rare diseases in our study, the majority of the cases responded well and had a complete recovery, but PRES may be the cause of death in some cases. Fortunately this did not happen to any of our cases yet Zama et al had $2.6 \%$ of their studied patients died due to PRES (11). While another study reported $11 \%$ of PRES patients developed rapidly progressive deterioration of neurological condition that resulted in death owing to PRES (14).

\section{Conclusion and Recommendations}

- In conclusion, PRES is more common in children with hematological malignancies in which MRI is the gold standard imaging modality for its diagnosis.

- The clinical and radiologic outcomes are reversible in most of the cases yet small number of patients will develop persistent brain damage or neurological deficit.

- Atypical PRES was more common in our study group than typical PRES.

- Patients who had atypical PRES location such as thalamic, brain stem, basal ganglia, deep white matter or diffuse frontal affection as well as patient who had areas of true restricted diffusion or post contrast enhancement showed a poor prognosis.

\section{References}

1.Federica S., Carol W., David S., ZieglerB., Glenn M., Marshall B.C., et al. (2014): 'Molecular profiling of childhood cancer': Biomarkers and novel therapies. BBA; Clinical 1: 59-77.

2. Kayahan E.M., Tore H.G., Bayrak A., Güngör D. and Coşkun M. (2009): 'MRI of central nervous system abnormalities in childhood leukemia'. Diagn interv Radio; 15: 86-92.

3. Vagace J.M., De la Maya , Caceres-Marzal C, Gonzalez de Murillo S. and Gervasini G. (2012): 'Central nervous system chemotoxicity during treatment of pediatric acute lymphoblastic leukemia/lymphoma'; 84: 276284.

4. Vázquez E., Lucaya J., Castellote A., Piqueras J., Sainz P., Olivé T.,et al. (2002): 'Neuroimaging in pediatric leukemia and 
lymphoma': Differential diagnosis RadioGraphics; 22: 1411-1428.

5. Vazquez E., Delgado L., Sanchwz-Montartez A., Barber I., Sánchez-Toledo J. and Enríquez G. (2011): 'Side effect of oncologic therapies in the pediatric central nervous system: Update in neuroimaging findings'. Radiographics; 31: 11231139.

6. Hinchey J., Chaves C., Appignani B., Breen J., Pao L., Wang A., et al. (1996): 'A reversible posterior leuckencephalopathy syndrome'. N Eng J Med1996; 334: 494-500.

7. Stevens C. J. and Heran M. K. (2012): The many faces of posterior reversible encephalopathy syndrome. The British Journal of Radiology; 85: 1566-1575.

8. Khan R.B., Sadighi Z. S., Zabrowski J., Gajjar A., and Jeha S. (2016): 'Imaging Patterns and Outcome of Posterior Reversible Encephalopathy Syndrome during Childhood Cancer Treatment', Pediatric Blood \& Cancer, 63(3), pp. 523-526.

9. Fugate J.E. and Rabinstein A.A. (2015): 'Posterior reversible encephalopathy syndrome: clinical and radiological manifestations, pathophysiology, and outstanding questions'. Lancet Neurology, 2015; 14(9): 914-925.

10. Hinduja A., Habetz K., Raina S., Ramakrishnaiah R. and Fitzgerald R.T. (2017): 'Predictors of poor outcome in patients with posterior reversible encephalopathy syndrome', International Journal of Neuroscience, 127(2), pp. 135-144.

11. Zama D., Gasperini P., Berger M., Petris M., De Pasquale , Cesaro S., et al. (2018): 'A survey on hematology-oncology pediatric AIEOP centres: The challenge of posterior reversible encephalopathy syndrome', European Journal of Haematology, 100(1), pp. 75-82.

12. Masetti R., Cordelli D.M., Zama D., Vendemini F., Biagi C., Franzoni E., et al.
(2015): 'PRES in Children Undergoing Hematopoietic Stem Cell or Solid Organ Transplantation', Pediatrics, 135(5), pp. 890 901

13. Siebert E., Bohner G., Endres M. and Liman T.G. (2014): 'Clinical and radiological spectrum of posterior reversible encephalopathy syndrome: does age make a difference? --A retrospective comparison between adult and pediatric patients.', PloS one, 9(12), p. e115073

14. Kamiya-Matsuoka C., Paker A.M., Chi L., Youssef A., Tummala S. and Loghin ME. (2016): 'Posterior reversible encephalopathy syndrome in cancer patients: a single institution retrospective study', Journal of NeuroOncology, 128(1), pp. 75-84.

15. Schweitzer A.D., Parikh N.S., Askin G., Nemade A., Lyo J., Karimi S., et al. (2017): 'Imaging characteristics associated with clinical outcomes in posterior reversible encephalopathy syndrome', Neuroradiology, 59(4), pp. 379-386.

16. Faille LD, Fieuws S. and Van Paesschen W. (2017): 'Clinical predictors and differential diagnosis of posterior reversible encephalopathy syndrome', Acta Neurologica Belgica, 117(2), pp. $469-475$.

17. Casey S.O., Sampaio R.C., Michel E. and Truwit C. L. (2000): 'Posterior Reversible Encephalopathy Syndrome: Utility of Fluidattenuated Inversion Recovery MR Imaging in the Detection of Cortical and Subcortical Lesions'. American Journal of Neuroradiology; 21: 1199-1206.

18. Yamamoto H., Natsume J., Kidokoro H., Ishihara N., Suzuki M., Tsuji T. et al. (2015): 'Clinical and neuroimaging findings in children with posterior reversible encephalopathy syndrome'. European Journal of Paediatric Neurology; 19(6): 672-678.

19. McKinney A.M., Short J., Truwit C.L., McKinney Z.J., Kozak O.S., SantaCruz K.S. et 
al. (2007): Posterior reversible encephalopathy syndrome: Incidence of atypical regions of involvement and imaging findings', American Journal of Roentgenology, 189(4), pp. 904-912.

20. Kidwell C. S., Alger J. R., Di Salle F., Starkman S., Villablanca P., Bentson J. et al (1999): 'Diffusion MRI in patients with transient ischemic attacks'. Stroke; 30(6): 1174-1180.

21. Donmez F. Y., Basaran C., Kayahan Ulu E. M., Yildirim M. and Coskun M. (2010): 'MRI Features of Posterior Reversible Encephalopathy Syndrome in 33 Patients'. Journal of Neuroimaging; 20: 22-28.

22. Bartynski W.S. and Boardman J.F. (2007): 'Distinct Imaging Patterns and Lesion Distribution in Posterior Reversible Encephalopathy Syndrome'. American Journal of Neuro Radiology; 28: 1320-1327.
23. Abdul-Qader I. A., Salwa T.I., Shaimaa A.M. (2017): 'MRI Features of Posterior Reversible Encephalopathy syndrome The Egyptian Journal of Hospital Medicine'; Vol.68 (2), Page 1229-12351229

24. Ni J., Zhou L.X., Hao H.L., Liu Q., Yao M., Li M.L. et al (2011): 'The Clinical and Radiological Spectrum of Posterior Reversible Encephalopathy Syndrome: A Retrospective Series of 24 Patients'. Journal of Neuroimaging; 21: $219-224$.

25. Roth C. and Ferbert A. (2013): 'The Posterior Reversible Encephalopathy Syndrome': What's Certain, What's New? Practical Neurology; 11: 136-144.

To cite this article: Medhat M. Refaat, Iman M. Zaky, Islam M. El Shazly, Mostafa M. Abd Al Gawad. Role of Magnetic Resonance Imaging in Detection and Follow up of Typical/atypical Posterior Reversible Encephalopathy Syndrome "PRES" in Pediatric Cancer Patients, BMFJ 2020; 37 (Radiology): 1-16, DOI: 10.21608/bmfj.2020.26775.1239 